\title{
Blühdorn, Ingolfur mit Butzlaff, Felix; Deflorian, Michael; Hausknost, Daniel; Mock, Mirijam (2020): Nachhaltige Nicht-Nachhaltigkeit. Warum die ökologische Transformation der Gesellschaft nicht stattfindet
}

\author{
Sybille Bauriedl \\ Eingegangen: 2. November 2020 - Angenommen: 2. Dezember 2020 - Online veröffentlicht: 3. Februar 2021
}

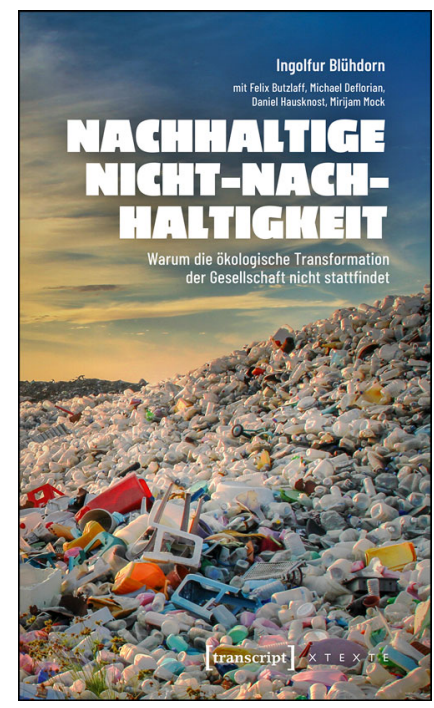

Der Begriff „Nachhaltigkeit“ erlebt aktuell eine Renaissance in der deutschsprachigen sozialwissenschaftlichen Umweltforschung. Seit nunmehr 50 Jahren wird die Dringlichkeit einer langfristigen Sicherung menschlicher Existenzgrundlagen - und menschlicher Existenz an sich - angemahnt. Die Studie „Grenzen des Wachstums“ (Meadows 1972) war nur ein Weckruf von sehr vielen. Die Fridays-for-Future-

Prof. Dr. Sybille Bauriedl, Interdisziplinäres Institut für Umwelt-, Sozial- und Humanwissenschaften, Europa-Universität Flensburg, Auf dem Campus 1, 24943 Flensburg sybille.bauriedl@uni-flensburg.de

\footnotetext{
(c) (1) () () 2021 Bauriedl; licensee oekom verlag. This Open Access article is published under the Creative Commons Attribution-ShareAlike 4.0 International Licence.
}

Bewegung brachte die immer umfangreicher vorliegenden Krisendiagnosen zurück auf die öffentliche Agenda und fordert die Nachhaltigkeitsziele Generationengerechtigkeit und globale Gerechtigkeit ein.

Ende der 1990er-Jahre hatte „Nachhaltigkeit“ als zentraler Analysebegriff der sozialwissenschaftlichen Umweltforschung und als normativer Begriff der Stadtentwicklung und Regionalplanung an Bedeutung verloren. Seitdem sind immer mehr Begriffe in die Umweltdebatte eingeflossen, die synonym verwendet werden. Die diskursmächtigsten Begriffe sind aktuell „,sozial-ökologische Transformation“, „Postwachstum“, „Grünes Wachstum“, ,,just transition“ und „Klimaneutralität“. Gemeinsam ist diesen Begriffen, dass sie die positive Utopie einer Welt mit Wohlstand für mehr Menschen ohne irreversible Umweltzerstörung aufrufen und einen Wandel hin zu emissionsminimierten Produktionsweisen für möglich halten. Die Wege zum Ziel unterscheiden sich jedoch sehr deutlich: Sie umfassen einen neuen Gesellschaftsvertrag, Gemeinwohlökonomie statt kapitalistischer Aneignung oder Entkopplung von Wirtschaftswachstum und Umweltverbrauch. Was diese Zukunftsvisionen von der Idee der Nachhaltigkeit unterscheidet, ist der zentrale Aspekt der globalen Gerechtigkeit als normativer Handlungs- und Wertorientierung. Das Spannungsfeld zwischen einer ökologischen Modernisierung (mit Effizienzgewinnen durch technologische Innovationen und Marktanreize) einerseits und einer strukturellen Ökologisierung (mit Suffizienzgewinnen durch starke Steuerung und qualitativem Wachstum) andererseits prägt die Nachhaltigkeitsdebatte der deutschen und europäischen Politik und Planung schon seit 30 Jahren.

Das Buch „Nachhaltige Nicht-Nachhaltigkeit“ macht den Stellenwert des Nachhaltigkeitsideals für die Gegen- 
wart deutlich, rekonstruiert den mittlerweile über 30-jährigen Weg der Nichtumsetzung dieses Ideals und gibt einen Zukunftsausblick. Die pessimistische Ausrichtung dieser Diagnose ist sofort offensichtlich. Autoren und Autorin haben einen Buchtitel gewählt, der den doppelten Wortsinn von nachhaltig im Sinne von dauerhaft und im Sinne eines Wandels zu mehr sozialer und ökologischer Gerechtigkeit spiegelt und im Untertitel ankündigt zu erklären, „,[w]arum die ökologische Transformation der Gesellschaft nicht stattfindet". Das Titelbild des Bandes repräsentiert eine entsprechende Dystopie gescheiterter Nachhaltigkeit: Ein riesiger Plastikmüllberg reicht bis zum Horizont.

Das Buch ist eine Monographie der Arbeitsgruppe des „Instituts für Gesellschaftswandel und Nachhaltigkeit“ an der Wirtschaftsuniversität Wien. Die zentralen Kapitel wurden vom Institutsleiter Ingolfur Blühdorn verfasst: Schlüsselkapitel zum Paradigmenwechsel der Nachhaltigkeitsdebatte, zur Diagnose einer Nicht-Nachhaltigkeit der Gegenwart sowie zum Demokratieproblem der NichtNachhaltigkeit. Ergänzt werden sie von Beiträgen seiner Mitarbeiterin und Mitarbeiter zu strukturellen Blockaden einer Nachhaltigkeitstransformation (Daniel Hausknost), Gestaltungsansprüchen politischer Parteien und Demokratisierungsbedarfen (Felix Butzlaff), dem Nischenaktivismus neuer sozialer Bewegungen (Michael Deflorian) und dem Ideal einer Konsumentenverantwortung (Mirijam Mock). Sie betrachten gesellschaftlich ausgehandelte Werte und Normen des Nachhaltigkeitsbegriffs aus soziologischer und politikwissenschaftlicher Perspektive und diagnostizieren, dass eine Gesellschaft der Nachhaltigkeit nicht in Sicht ist - und dass diese zu propagieren ein Teil des Problems sei. Blühdorns Anliegen als Nachhaltigkeitssoziologe ist es ganz offensichtlich nebenbei auch, einen Kontrapunkt zum Buch „Gesellschaft der Nachhaltigkeit“ des Soziologen Sighard Neckel und seiner Hamburger Forschungsgruppe „Zukünfte der Nachhaltigkeit“ zu setzen, das ebenfalls im transcript-Verlag erschienen ist (Neckel/Besedovsky/ Boddenberg et al. 2018).

Blühdorn bezeichnet seine Beschreibung der Nachhaltigkeitsdebatte in Deutschland als Gegenwartsdiagnose: Er nimmt den Übergang von einem hoffnungsvollen Nachhaltigkeitsideal der Vergangenheit, das eine Ökologisierung durch demokratische Entscheidungen und vernunftgetriebenes Handeln erzielen wollte, hin zu einer postdemokratischen, expertokratischen Verbotskultur der Gegenwart wahr. Für ihn stehen die beschleunigte Zunahme der Umweltverschmutzung der letzten Jahrzehnte und die „Konjunktur“ rechtspopulistischer Bewegungen und nichtnachhaltiger Lebensstile in einem direkten Zusammenhang (S. 66). Diesen Befund benutzt er als Beweis für die Untauglichkeit des Demokratie- und Modernisierungsmodells der Industriegesellschaften, die keine Entkopplung von
Wirtschaft und Ressourcenverbrauch bewirken konnten. Auch die Klimaforschung, ökosoziale Bewegungen und Fridays for Future haben laut Blühdorn der Nicht-Nachhaltigkeit nichts entgegenzusetzen, schlimmer noch: Sie hätten diese verschärft, indem sie Angst vor Enteignung und Entfremdung schürten. Mit der Übernahme der bewegungspolitischen Belange durch sozialwissenschaftliche Klimaforscherinnen und Klimaforscher sei Klimaschutz zur Bevormundungspolitik geworden (S. 78), die einen Rechtsruck befördere und Wahrheitsverweigerer und Klimaleugner produziere (S. 80). Die Rhetorik von Dringlichkeit und Notstand in der Klimadebatte bezeichnet Blühdorn als Strategie einer autoritären Agenda, die er mit der Migrationsrhetorik von Rechtspopulisten vergleicht. Die Aufklärerinnen und Aufklärer wären damit Schuld an dem Entstehen der Reaktionären.

Diese Argumentationslinien bleiben frei von empirischen Belegen und basieren auf verkürzten und teilweise verdrehten Kausalitäten von Ursachen und Wirkung. Blühdorn postuliert, dass neben wissenschaftlichen Fakten die Relevanz subjektiver Wahrheiten stärker zu berücksichtigen sei, nachdem die ökosozialen Bewegungen unintendiert „zur Destabilisierung der objektiven Wahrheit" beigetragen hätten (S. 82). Ausgerechnet die Wissenschaftlerinnen und Wissenschaftler der sozialökologischen Transformationsforschung werden von Blühdorn verantwortlich gemacht für eine nachhaltige Nicht-Nachhaltigkeit: Sie hätten die Geschichte des ökologischen Aufbruchs weitererzählt, um an Fördertöpfen zu partizipieren und Denkverbote gegen Zweifler einer Postwachstumsgesellschaft zu pflegen (S. 24 f.). Sie schränkten damit angeblich die liberale Demokratie ein, die Blühdorn als größte Errungenschaft moderner Gesellschaften und Grundbedingung von Nachhaltigkeit versteht.

Blühdorns Rezept für mehr Nachhaltigkeit ist dementsprechend eine kritische Haltung gegenüber den ,,alle Objektivität monopolisierenden Expert*innen“ (S. 95). Damit sind explizit das IPCC (Intergovernmental Panel on Climate Change) und der WBGU (Wissenschaftlicher Beirat der Bundesregierung Globale Umweltveränderungen) gemeint, deren Forderungen „,von wesentlichen Teilen der Gesellschaft als elitär und statusbedrohend wahrgenommen werden" (S. 95). Es verwundert sehr, dass ein Soziologe derart unbestimmt über, Gesellschaft" spricht, die von rechts angegriffene wissenschaftliche Objektivität nicht differenziert bzw. verteidigt und die gesellschaftstheoretischen und methodischen Zugänge seiner Gegenwartsdiagnose nicht benennt. An vielen Textstellen ist nicht zwischen Beschreibung, Diagnose und Haltung des Autors zu unterscheiden. Er ignoriert zudem die zunehmende Fragmentierung von Gesellschaften seit den 1990er-Jahren sowie zentrale Erkenntnisse zur Bedeutung sozialer Kategorien wie Klasse, 
Geschlecht und kultureller Herkunft in der Nachhaltigkeitsforschung.

In der Soziologie sind Gegenwartsdiagnosen ein verbreitetes Genre. Im Idealfall betrachten sie Sachverhalte im Rückblick, bewerten diese neu und bieten eine möglichst realistische Einschätzung wahrscheinlicher Entwicklungen. Für eine Vertiefung der vorliegenden Diagnose einer anhaltenden Nicht-Nachhaltigkeit sollten die Schnittstellen von Generationengerechtigkeit, sozialer Gerechtigkeit und globaler Gerechtigkeit stärker in den Fokus gerückt werden, um die fundamentalen Zielkonflikte, divergenten Leitbilder und Gerechtigkeitsvorstellungen für die Gegenwart und Zukunft einer nachhaltigen Gesellschaft differenzierter verstehen zu können. Das Buch zeigt, dass die Soziologie der Nachhaltigkeit ein aktives Forschungsfeld mit sehr kontroversen Positionen und Betrachtungsweisen ist. Es bleibt zu hoffen, dass hieraus weiterführende, konstruktive Debattenbeiträge für eine nachhaltige, lebensbewahrende Zukunft entstehen.

\section{Vollständige bibliographische Angaben des rezensierten Werkes:}

Blühdorn, Ingolfur mit Butzlaff, Felix; Deflorian, Michael; Hausknost, Daniel; Mock, Mirijam (2020): Nachhaltige Nicht-Nachhaltigkeit. Warum die ökologische Transformation der Gesellschaft nicht stattfindet. Bielefeld: transcript-Verlag. 330 Seiten.

\section{Literatur}

Meadows, D. (1972): Die Grenzen des Wachstums. Bericht des Club of Rome zur Lage der Menschheit. Stuttgart.

Neckel, S.; Besedovsky, N.; Boddenberg, M.; Hasenfratz, M.; Pritz, S. M.; Wiegand, T. (Hrsg.) (2018): Die Gesellschaft der Nachhaltigkeit. Umrisse eines Forschungsprogramms. Bielefeld. 